\title{
Biotransformation of Furaltadone, Furazolidone and Nitrofurazone using Aspergillus Tamarii isolate TN-7 : in Vitro Residual Identification and Quantification by HPLC-DAD
}

\author{
N S Mohammad ${ }^{1}$, M I A Halim ${ }^{2}$, M M Mahat ${ }^{3}$, M F Safian' ${ }^{2}$ Z Z Ariffin ${ }^{1 *}$ \\ ${ }^{I}$ School of Biology, Faculty of Applied Sciences, Universiti Teknologi MARA, 40450 Shah Alam, Selangor, Malaysia \\ ${ }^{2}$ School of Chemistry and Environment, Faculty of Applied Sciences, Universiti Teknologi MARA, 40450 Shah Alam, Selangor, \\ Malaysia \\ ${ }^{3}$ School of Physics and Material Studies, Faculty of Applied Sciences, Universiti Teknologi MARA, 40450 Shah Alam, Selangor, \\ Malaysia \\ *Corresponding author Email: drzaidah@salam.uitm.edu.my
}

\begin{abstract}
Nitrofurans (NFs) such as furaltadone (FTD), furazolidone (FZD) and nitrofurazone (NFZ) have been used as antibacterials and growth promoters for the poultry and aquaculture industry. These antibiotics have now been banned from use due to their carcinogenic properties; therefore there is an urgent need to remove or degrade NFs from contaminated areas. Aspergillus tamarii isolate TN-7 isolated from antibiotic overexposed soil shows an ability to degrade the NFs antibiotics. After 5 days of incubating of Aspergillus tamarii isolate TN-7 with $500 \mu \mathrm{g} / \mathrm{mL}$ NF, the residual of the NF concentration was determined by High-Performance Liquid Chromatography-diode array detector (HPLC-DAD). Solid phase extraction was performed to clean-up the fermentation broth prior to HPLC-DAD analysis. Antimicrobial of the NFs residues showed a decreased in the percentage of inhibition that FTD, FZD and NFZ were reduced to to $85.71 \%, 75.86 \%$ and $70.97 \%$ after 96 hours of incubation. Quantification using HPLC-DAD showed, after 96 hours of incubation, Aspergillus tamarii isolate TN-7 reduced furaltadone, furazolidone and nitrofurazone to $86.73 \%, 37.49 \%$ and $29.17 \%$ respectively. This finding shows that Aspergillus tamarii isolate TN-7 has the potential to be used as a bioremediation tool in removing $\mathrm{NF}$ antibiotics from the contaminated areas.
\end{abstract}

Keywords: Aspergillus tamarii isolate TN-7; biotransformation; degradation; HPLC-DAD; nitrofuran.

\section{Introduction}

This Nitrofurans (NFs) such as furaltadone (FTD), furazolidone (FZD) and nitrofurazone (NFZ) are widely used in veterinary drugs for poultry and aquaculture. NFs are also used to treat disease related to gastrointestinal tract and common disease by bacteria and protozoa by inhibiting microbial enzymes that involved in carbohydrate metabolism [1]. NFs have since been banned and prohibited from usage in poultry or livestock production. The U.S. Food Drug and Administration (FDA) ban imports that have NF residues in them. The European Union (EU) have banned the usage of NFs in livestock production since 1995 because of their carcinogenic and toxic effects on humans [2]. NF parent drugs are unstable and are rapidly metabolized to their metabolites (NFMs), FTD (AMOZ; 3-amino-5-morpholinomethyl-2-oxazolidinone), FZD (AOZ; 3-amino-2-oxazolidinone) and NFZ (SEM; semicarbazide). These NFMs maintain their stability when bound to tissue or protein which can cause toxic and carcinogenic effects. NFs administered on the poultry will be excreted through faeces or urine of the chickens. These will enter or will be introduced to soil

environment and cause soil contamination. According to Yu et al., [3], administration of NF in aquaculture will also cause the fish to excrete the NFs or NFMs that then will enter the aquatic environment and cause antibiotic contamination. Agriculture of contaminated soil and aquatic environments exposed to these antibiotics will then take up the NFs and NFMs, which will then be transferred deleterious effects to humans. Continuous deposition of antibiotics into the environment can increase the resistance of bacteria towards these antibiotics. Thus, there is an urgent need to degrade these antibiotics that have been deposited into the environment. Aspergillus sp. is a species of fungus that can be commonly found in soil [4] that showed great potential in degrading pollutant. Aspergillus tamarii were reported to have the ability to degrade endosulfan sulfate [5] and dyes [6]. The aim of this study is to determine the ability of the Aspergillus tamarii isolate TN-7 to degrade nitrofuran antibiotics.

\section{Experimental}

\subsection{Reagents and chemicals}

Analytical grade ethyl acetate (EtOAc $>99.95 \%)$, was purchased from Bendosen, HPLC grade methanol $(\mathrm{MeOH}>99.90 \%)$ from Systerm, water HPLC grade was purchased from Amresco, and acetonitrile (ACN > $99.90 \%$ ) was purchased from Merck. Nitrofurans antibiotics, furaltadone $\left(\mathrm{C}_{13} \mathrm{H}_{16} \mathrm{~N}_{4} \mathrm{O}_{6}\right)$, furazolidone $\left(\mathrm{C}_{8} \mathrm{H}_{7} \mathrm{~N}_{3} \mathrm{O}_{5}\right)$ and nitrofurazone $\left(\mathrm{C}_{6} \mathrm{H}_{6} \mathrm{~N}_{4} \mathrm{O}_{4}\right)$ were purchased from Sigma-Aldrich. 


\subsection{Materials}

The SPE clean-up column, HyperSep C18 SPE column, $3 \mathrm{~mL}$ with $500 \mathrm{mg}$ bed weight was purchased from, Thermo Fisher Scientific. The analysis was made using Agilent Technologies 1200 series, with reverse phase $\mathrm{C} 18$ Agilent analysis column (ZORBAX SB-C18, $5 \mu \mathrm{M}, 4.6 \times 250 \mathrm{~mm}$ ) and the mobile phase consisting of water and acetonitrile with detection at $365 \mathrm{~nm}$. The ChemStation for LC 3D (Rev. A. 10.02) software was used to control the equipment and analyze the output data.

\subsection{Standard solution preparation}

FTD, FZD and NFZ stock standard solutions were prepared at a concentration of $1000 \mathrm{ppm}$ using acetonitrile. The stocks were covered with aluminium foil and kept at $4{ }^{\circ} \mathrm{C}$ for a maximum of a 1 month. Working standards were prepared freshly prior to analysis.

\subsection{Soil sample collection}

The soil sample was collected at a soil plot where veterinary antibiotics have been used extensively from Ayu Farm, Meru, Klang with a GPS coordinate of N3.093219 E101.40269. A weight of $500 \mathrm{~g}$ of soil was collected from a depth of $15 \mathrm{~cm}$. Soil samples collected and preserved in sterile plastic bags and before being stored in an icebox to maintain the soil's natural properties. The soil was then taken to the laboratory and stored at $4{ }^{\circ} \mathrm{C}$.

\subsection{Identification of isolated soil fungi}

\subsubsection{Morphological and molecular identification}

The isolated soil fungi were identified using morphological and molecular characterization. Morphological characteristics are based on microscopic and macroscopic observations. For microscopic observation, fungi were stained with methylene blue solution and viewed using $1000 \mathrm{X}$ magnification under a light microscope to observe for fungi features such as hyphae and conidia. Macroscopic characterization with the naked eyes was performed, to characterize based on colony growth including length and growth, presence or absence of mycelium, pigment production, colour and wrinkle furrow [8].

\subsubsection{Molecular identification}

DNA was extracted using DNA extraction kit, innuPREP Plant DNA kit from Analytic Jena (Germany) as per the manufacturer's instructions. The DNA extracted was amplified using $0.2 \mu \mathrm{M}$ ITS 1 (5'-TTC GTA GGT GAA CCT GCG G-3') forward primer and $0.2 \mu \mathrm{M}$ ITS 4 (5'-TCC TCC GCT TAT TGA TAT GC-3') reverse primer [7]. PCR master mix was prepared using Top Taq DNA polymerase by QIAGEN which contained 10x TopTaq PCR buffer, Coralload concentrate, Q-solution, dNTP mix and TopTaq DNA polymerase. PCR conditions were initial denaturation $\left(94{ }^{\circ} \mathrm{C}\right.$ for 3 minutes), 30 cycles of denaturation $\left(94{ }^{\circ} \mathrm{C}\right.$ for 30 seconds), annealing $\left(61.4{ }^{\circ} \mathrm{C}, 30\right.$ seconds), extension $\left(72{ }^{\circ} \mathrm{C}, 1\right.$ minute) and final extension $\left(72{ }^{\circ} \mathrm{C}, 5\right.$ minutes $)$.

\subsection{Biotransformation assay}

Fermentation was performed according to Dong et al., [8] with some modifications using a Potato Dextrose Broth (PDB). The broth was divided equally $(100 \mathrm{~mL})$ into 4 different $250 \mathrm{~mL} \mathrm{Er}$ lenmeyer flasks. A mycelia agar plug, $2 \mathrm{~mm}$ in size was inoculated into each culture bottle accordingly. PDB with culture only and PDB with nitrofurans only were used as controls. All culture bottles were incubated and then agitated at a fixed $120 \mathrm{rpm}$ at $25^{\circ} \mathrm{C}$ $\log$ phase was reacted. All experiments were carried out in triplicates.

After the fungus growth has reached the respected exponential phase, $10 \mathrm{~mL}$ volume of nitrofuran antibiotics, furaltadone, furazolidone and nitrofurazone was added into the culture flask to give a final concentration of $500 \mu \mathrm{g} / \mathrm{mL}$. The flasks were then incubated for another 96 hours at $120 \mathrm{rpm}$ and $25^{\circ} \mathrm{C}$. Every 24 hours, $6 \mathrm{~mL}$ of the medium is withdrawn as biotransformation products and the $\mathrm{pH}$ of fermentation broth recorded. All the biotransformation products were stored at $4{ }^{\circ} \mathrm{C}$ prior to extraction of the nitrofuran antibiotic residual.

\subsection{Liquid-liquid extraction (LLE)}

Every 24 hours of incubation, the biotransformation products are subjected to liquid-liquid extraction. This extraction technique was done according to Haller et al., [9] and was carried out in a separating funnel. All samples were mixed with an extraction solvent, ethyl acetate (EtOAc). A volume of $25 \mathrm{~mL}$ of EtOAc and $25 \mathrm{~mL}$ of distilled water were added into the separating funnel together with $6 \mathrm{~mL}$ of the biotransformation products. Extraction of the nitrofuran antibiotic residual was carried out by shaking the sample with EtOAc and distilled water for two minutes with periodic venting out of the air. The phase separation process was then allowed to take place. After 10 to 15 minutes, the lower organic layer was put through the same process twice by adding the same volume of EtOAc from the first extraction earlier for each process. All extractions collected were combined and concentrated using a rotary evaporator. $500 \mu \mathrm{L}$ of DMSO was used to mix the concentrated nitrofuran residual and then stored in a microcentrifuge tube at $4{ }^{\circ} \mathrm{C}$.

\subsection{Clean-up of antibiotics residual}

Solid-phase extraction (SPE) was done according to Jewell et al., [10]. A volume of $3 \mathrm{~mL}$ methanol and $3 \mathrm{~mL}$ water (HPLC grade) were used to condition the SPE column. A volume of $4 \mathrm{~mL}$ of the sample was added to the column cartridges. The samples were washed using $4 \mathrm{~mL}$ of HPLC grade water and then eluted with 4 $\mathrm{mL}$ methanol. Samples (volume of $1 \mathrm{ml}$ ) were fractionally eluted for each tube. This is to ensure that dimethylsulfoxide (DMSO) and other contaminants were eluted and did not combine with the antibiotic compounds. Then the second tube and third tube of the eluted compound were optimized such that they showed the highest amount of nitrofuran antibiotics when injected into the HPLCDAD system. The second and third tube of the eluted compound were combined and used for the HPLC-DAD analysis. The eluted compound was then diluted with acetonitrile at a 1:10 dilution and filtered using a $0.45 \mu \mathrm{M}$ syringe filter before being injected into the HPLC-DAD system

\subsection{Antimicrobial assay of nitrofuran residues}

The antimicrobial activity of the nitrofuran residual was screened against Escherichia coli ATCC 25922, Staphylococcus aureus ATCC 25923, Salmonella typhi ATCC 14028 and Streptococcus pyogene ATCC 19615 in triplicates. These bacteria were selected because they are normally susceptible to nitrofuran antibiotics. The antimicrobial assay of the selected bacteria was carried out by using well diffusion techniques [11] with some modifications [12]. Overnight cultures were prepared in a Mueller-Hinton broth and diluted to $10^{8} \mathrm{CFU} \mathrm{ml}{ }^{-1}$, which corresponds to $0.5 \mathrm{McF}$ arland standards. Sterile cotton swabs were used to spread the culture over the surface of the Mueller Hinton agar plates in triplicates and allowed to dry. Five wells were made on agar plates using a sterile cork borer. One well was reserved for sterile DMSO as the negative control. Another well was reserved for a clindamycin antibiotic with a concentration of $40 \mu \mathrm{g} / \mathrm{mL}$ as the positive control and one well for the abiotic control. The zones of inhibition pro- 
duced by each well were measured in three directions around the well and the mean diameter was recorded.

\subsection{Quantification of nitrofuran residual by HPLC- DAD}

The HPLC-DAD analysis was performed according to Wang and Zhang [13] which suggested that HPLC grade water and acetonitrile were the best mobile phase for the analysis of nitrofuran antibiotics. For this study, the mobile phase A consist of water and the mobile phase B is acetonitrile. The isocratic mobile phases A and $\mathrm{B}$ were combined at a ratio of $40 \%$ and $60 \%$, respectively, for furaltadone, A flow rate of $1.0 \mathrm{ml} / \mathrm{min}$ was maintained and $10 \mu \mathrm{L}$ of furaltadone residues injected into the system. For furazolidone and nitrofurazone, mobile phases $\mathrm{A}$ and $\mathrm{B}$ were combined with a ratio of $50 \%$ and $50 \%$, respectively, at a flow rate of $1.2 \mathrm{ml} / \mathrm{min}$ and $20 \mu \mathrm{L}$ of compounds injected into the system. The HPLCDAD analysis was carried out at $365 \mathrm{~nm}$ detection.

\section{Results and discussion}

\subsection{Morphological and molecular identification of Aspergillus tamarii isolate TN-7}

Aspergillus tamarii isolate $\mathrm{TN}-7$ is a yellow to green coloured colony. It was observed to be in circular and powdery form. The colonies diameter was $8.4 \mathrm{~cm}$ after four days of incubation. No colour change was observed after 14 days of incubation. Microscopic showed long conidiophores, vesicle attached to phialides and the end of it was conidiospores. DNA sequence of the Aspergillus tamarii isolate TN-7 was analyzed using BLAST (NCBI) and the percentage identity to known fungal sequences in the GeneBank database (NCBI) was $99 \%$ identity and accession number of KX610719.1.
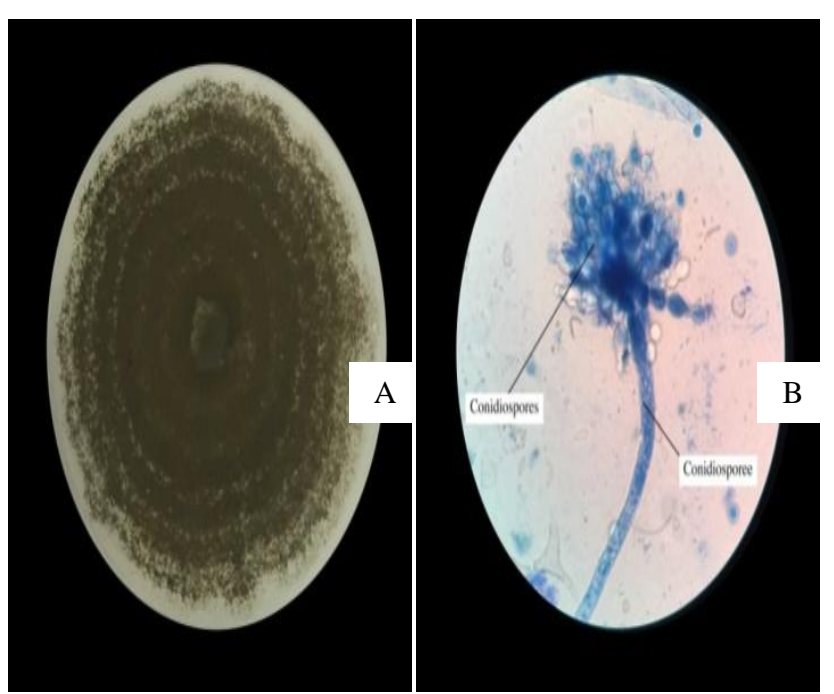

Fig. 1: Aspergillus tamarii isolate TN-7. A) Macroscopic view after 4 days of incubation. B) Microscopic view after 3 days of incubation at $1000 \mathrm{X}$ magnification.

\subsection{Antimicrobial assay of nitrofuran residual}

Percentage of inhibition was calculated by the diameter of the nitrofurans residues inhibition zone over the diameter of the abiotic control inhibition zone. The diameter of abiotic control served as a control, where no degradation of nitrofurans antibiotic occurred. Fig. 2 shows the antimicrobial activity of nitrofurans residues with selected bacteria.
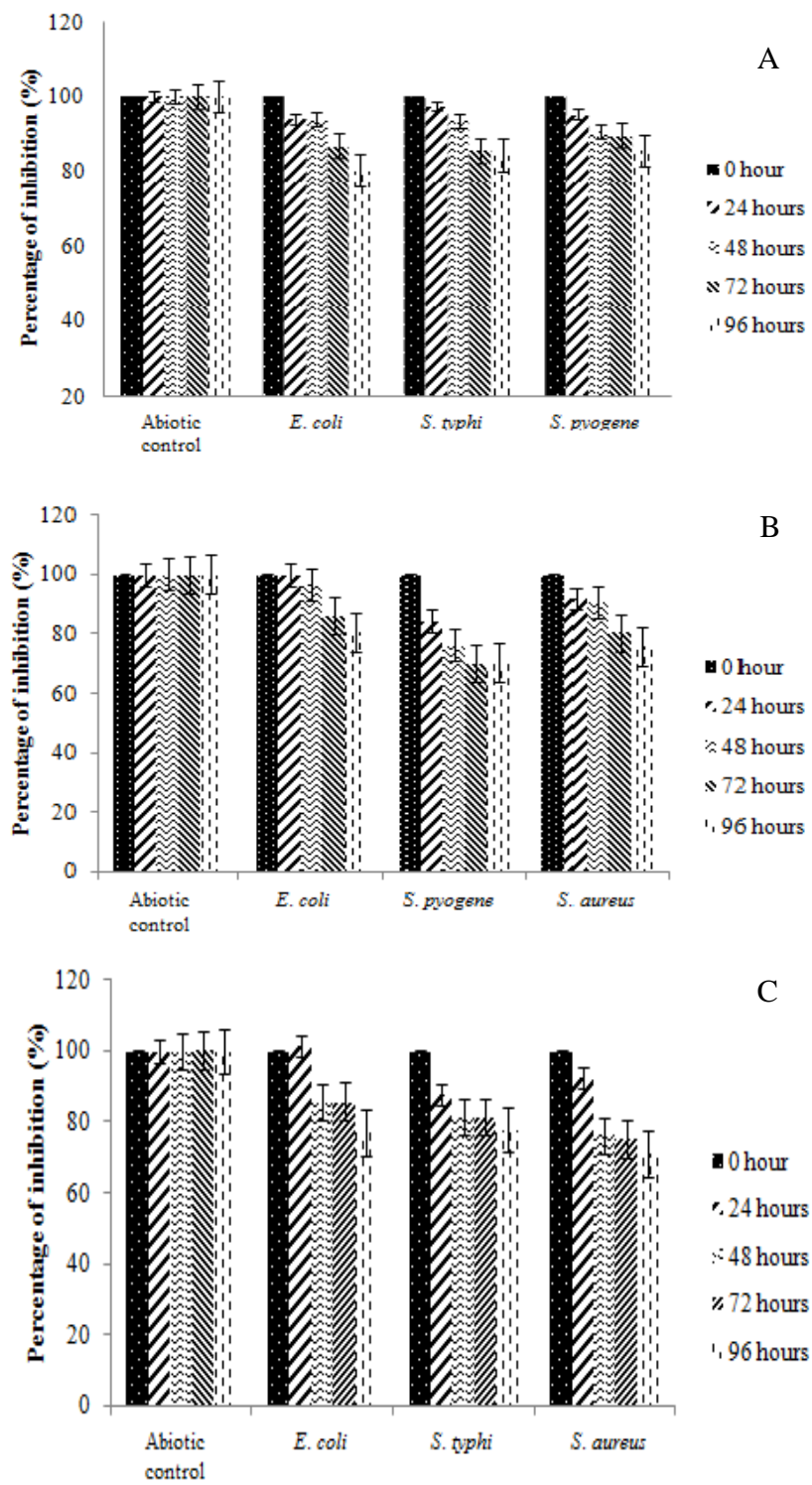

Fig. 2: Antimicrobial of nitrofurans residues. A. Furaltadone residues; B Furazolidone residues and C. Nitrofurazone residues. The error bars represent standard error of the mean and run in triplicates with significantly reduced the antibacterial activity at $(p<0.05)$.

Degraded or transformed antibiotic would lose its function as antibacterial as the chemical structure of the antibiotic was modified or degraded. Degradation of the antibiotics leads to a change in the antibiotic properties as antibacterial agents. Under certain conditions, the degraded compounds may have better antibacterial properties than the parent drugs. From the plotted graph in (Figure 2), after 96 hours of incubation, the antibiotic residues showed a decrease in antibacterial activities towards the selected bacteria. Levison and Levison [14] reported that the low antibacterial activity of antibiotic residues was due to the low concentration of antibiotic residues degraded by Aspergillus tamarii isolate TN-7.

\section{$3.3 \mathrm{pH}$ of the fermentation broth}

After 96 hours of incubation, the $\mathrm{pH}$ of the fermentation broth containing Aspergillus tamarii and nitrofurans antibiotics was observed to increase (Fig. 3). Aspergillus tamarii isolate TN-7 was isolated from soil with a $\mathrm{pH}$ of 6.8. For Aspergillus tamarii isolate $\mathrm{TN}-7$ as the mycelia mass increased the $\mathrm{pH}$ of the media also increased. According to Dariush [15], changes in the $\mathrm{pH}$ medium can shift the metabolite particularly when the targeted product is an organic acid. High production of fungal mycelia mass significantly found to decrease or increase the $\mathrm{pH}$ medium in some fungi. 


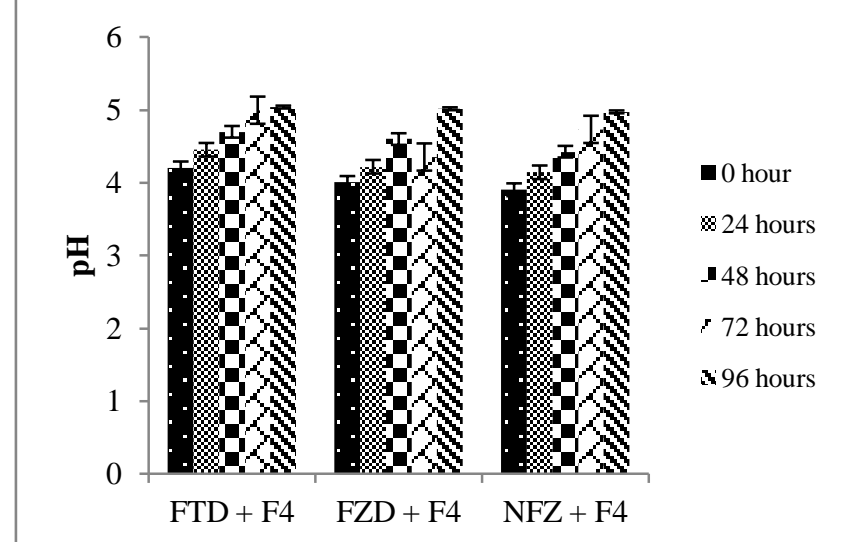

Fig. 3: $\mathrm{pH}$ value of fermentation media. FTD = furaltadone; FZD = furazolidone; $\mathrm{NFZ}=$ nitrofurazone; $\mathrm{F} 4=$ Aspergillus tamarii isolate $\mathrm{TN}-7$

\subsection{HPLC-DAD analysis}

FTD, FZD and NFZ standards were eluted at $3.86 \mathrm{~min}, 3.62 \mathrm{~min}$ and 2.46 min respectively. Standards curves of the NFs showed good linearity $\left(\mathrm{R}^{2}>0.99\right)$ and can, therefore, be used to quantitate the NF residues. Initially, $20 \mu \mathrm{L}$ of the FTD was injected into the system with A to B mobile phase ratio of $50: 50$ at $1.2 \mathrm{ml} / \mathrm{min}$ flow rate, resulting in a broad, fronting and split peak. This was then optimised such that the injection volume was reduced to 10 $\mu \mathrm{L}$, with a mobile phase ratio of $40: 60$ and a flow rate of 1.0 $\mathrm{ml} / \mathrm{min}$ that produced sharper peak and eluted faster. Increased acetonitrile caused the peak to elute faster. Wang and Zhang [13], reported that a higher ratio of organic solvents could also shorten the retention time.
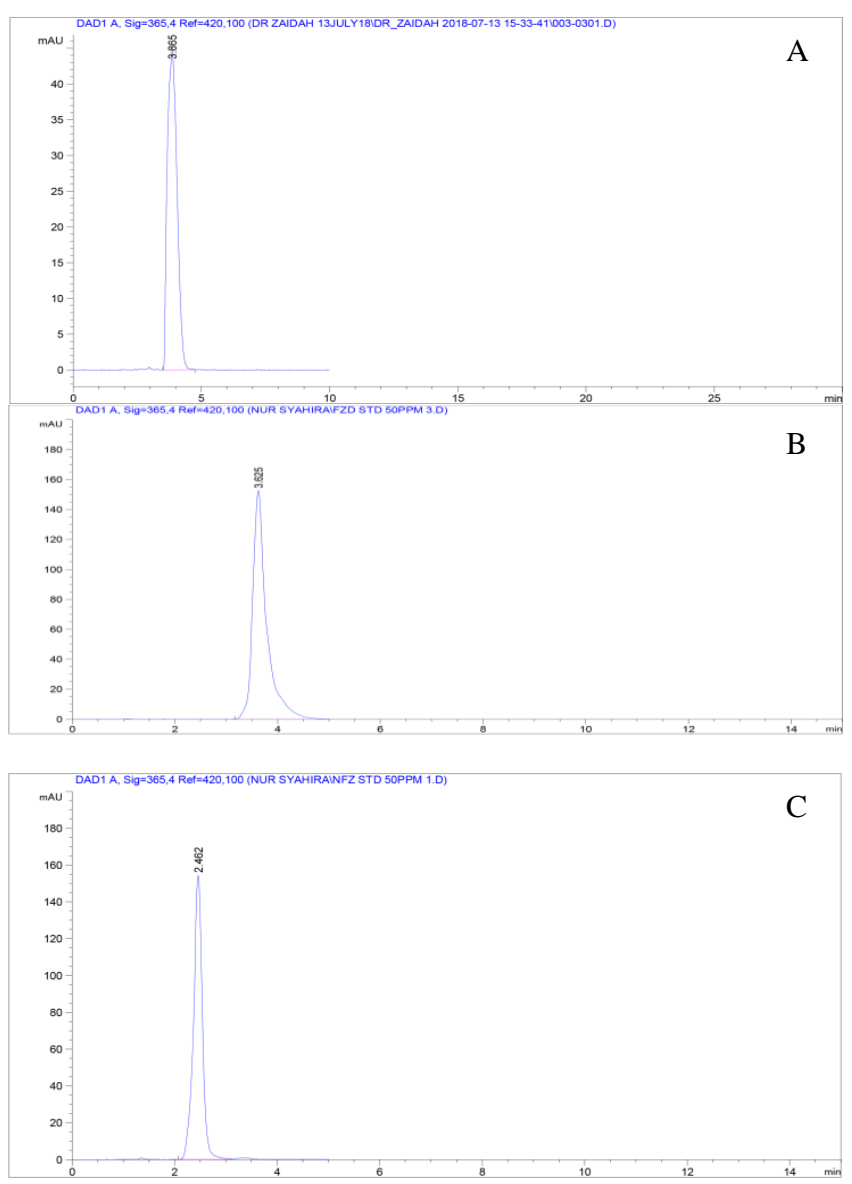

Fig. 4: HPLC chromatogram of the nitrofurans standards (50 ppm) with mobile phase composition of (water : acetonitrile). A) FTD, furaltadone, mobile phase $(40: 60)$; B) FZD, furazolidone, mobile phase $(50: 50)$ and C) NFZ, nitrofurazone, mobile phase $(50: 50)$.
From the HPLC-DAD analysis, the concentration of NFs in Fig. 5 reduced as the incubation time increased. Furaltadone was reduced to $86.73 \%$ after being incubated for 96 hours with Aspergillus tamarii isolate TN-7. Single factor ANOVA conducted showed that the data obtained was significant as the $p$-value is $p<0.05$. Aspergillus tamarii isolated TN-7 showed good degradation ability towards FZD and NFZ which were reduced to $37.49 \%$ ( $p=$ $0.024)$ and $29.17 \%(p=0.025)$, respectively. Chromatogram of nitrofurazone residues (Fig. 6) showed DMSO peak was eluted at retention time 1.611 min which supported in the standard that reported by Agilent Technologist, [16] DMSO that used in sample preparation eluted around retention time at $1.5 \mathrm{~min}$. Peaks that were eluted at $1.93 \mathrm{~min}, 2.07 \mathrm{~min}$ and $3.35 \mathrm{~min}$ were most probably the metabolites formed. However, in this study, the metabolites formed remained unknown as further analysis using LC-MS is required.

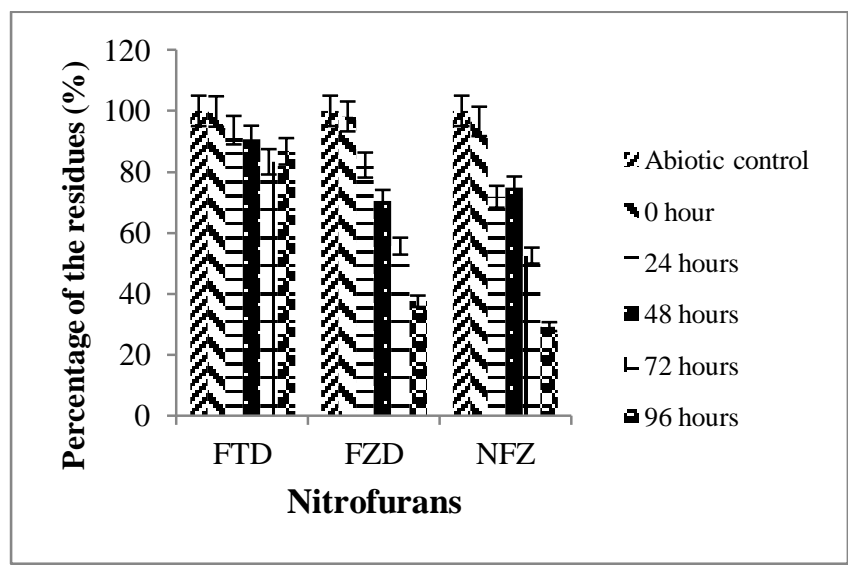

Fig. 5: Percentage residues of nitrofurans antibiotics, FTD (furaltadone); FZD (furazolidone) and NFZ (nitrofurazone).

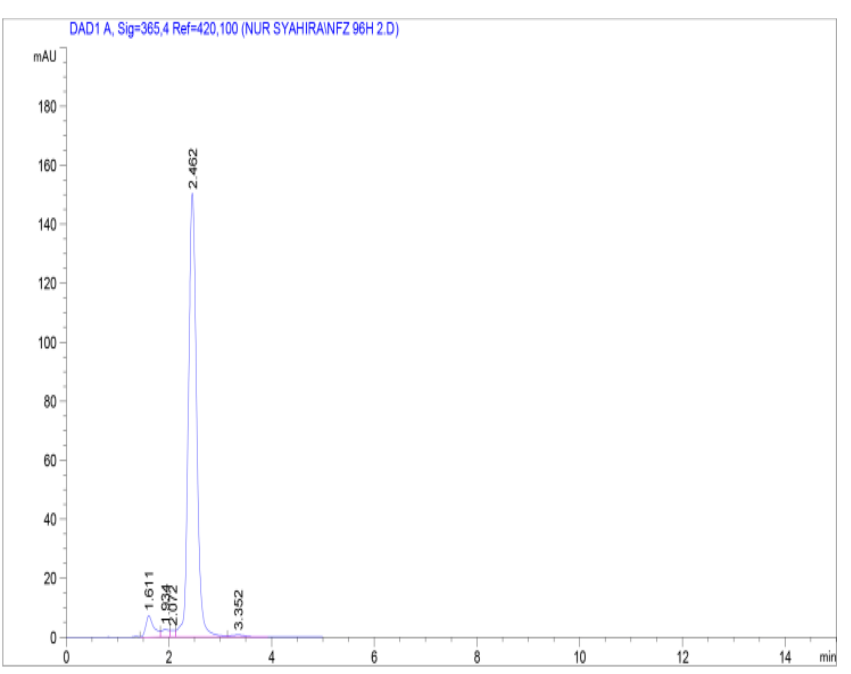

Fig. 6: Chromatogram of nitrofurazone residues (NFZ) after 96 hours of incubation at a flow rate of $1.2 \mathrm{~mL} / \mathrm{min}$.

According to Nouws et al., [17], there are two main degradation pathways of the nitrofuran (Fig. 7), one is in the 5-nitrofuran ring and the other is at the side chain $\mathrm{R}$ where the metabolites are bound. The side chain $\mathrm{R}$ is susceptible to deacetylation and oxidation. At the nitrofuran ring, the $\mathrm{NO}_{2}{ }^{-}$is more susceptible to reduction occur. Nitroreductase enzyme present in bacteria and nitroreductase-like proteins that are present in eukaryotes and archaea have the ability to reduce the nitrofuran ring. 5-nitrofurans may undergo one-electron and two-electron reductions the European Food Safety Authority [18]. There are two types of nitroreductases (Type I and II). Type I nitroreductase are expressed in bacteria and eukaryotes catalyse two-electron reduction while Type II mediates the one-electron reduction in most mammalian tissue. 
(A)

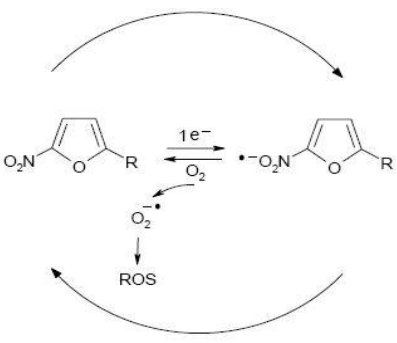

(B)

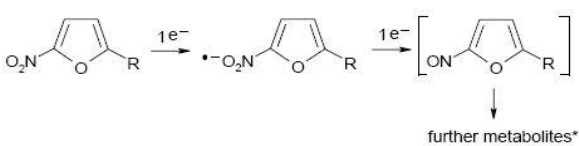

Fig. 7: Nitroreduction of 5-nitrofuran ring. A) one-electron reduction and B) two-electron reduction [18].

The one-electron reduction was found to occur in aerobic condition where the nitro anion radical is formed. The nitro anion radical can be oxidised back to the parent compound to produce superoxide anion, which generates Reactive Oxygen Species (ROS) [19, 20]. Two-electron reduction further reduced to a more stable hydroxylamine group and further metabolized to form three compounds, 1) a stable amine group, 2) nitrenium ion and unsaturated and saturated open chain nitriles (Fig. 8).

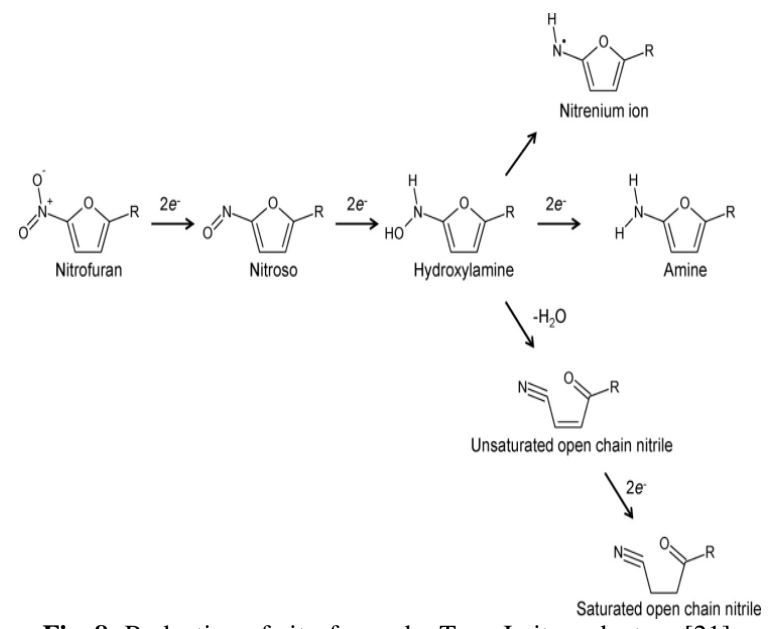

Fig. 8: Reduction of nitrofurans by Type I nitroreductase [21]

Some of the factors that affect antibiotic degradation are $\mathrm{pH}$, temperature and agitation rate. Different $\mathrm{pH}$ ranges produce different degradation products. The nitrofurantoin structure that is also similar to the furazolidone structure and is more unstable as the $\mathrm{pH}$ increases and degrades into more compounds when compared to acid hydrolysis [22]. Besides, a high agitation rate creates an energy supply for mixing and transferring oxygen that grows and freely disperses [15]. The higher the agitation rate, the higher the mycelia biomass produced, as the mixed oxygen supply transfers sufficient oxygen for the growth. The higher the aeration in the fermentation media, the more mycelia mass will be produced. as the oxygen supply for growth and degradation are in a sufficient amount. However high agitation rate can damage the mycelia.

\section{Conclusion}

In conclusion, Aspergillus tamarii isolate $\mathrm{TN}-7$ can be used as an application for treating soil or aquatic environments that have been contaminated with NF antibiotics. Further analysis using LCMS is required to determine the metabolites formed from the biotransformation activity.

\section{Acknowledgement}

This work is supported by Geran Inisiatif Penyeliaan (GIP), 600IRMI/GIP 5/3 (0059/2016) and Bestari, 600-IRMI/DANA 5/3 BESTARI (P) (010/2018).

\section{References}

[1] Leston S, Nunes M, Viegas I, Lemos MFL, Freitas A, Barbosa J, Ramos F, \& Pardal MA, (2011), The effects of the nitrofuran furaltadone on Ulva lactuca. Chemosphere, 82(7), 1010-1016.

[2] Commission Regulation, Annexes I, II, III and IV to Regulation (EEC) No 2377/90 laying down a community procedure for the establishment of maximum residue limits of veterinary medicinal products in food stuffs of animal origin, C Regulation, et al., Editors. 1995, Official J. Eur. Communities.

[3] Yu WH, Chin TS, \& Lai HT, (2013), Detection of nitrofurans and their metabolites in pond water and sediments by liquid chromatography (LC)-photodiode array detection and LC-ion spray tandem mass spectrometry. International Biodeterioration \& Biodegradation, 85, 517-526.

[4] Senwan MS, Safian MF, Noor ZM, \& Ariffin ZZ, (2017), Isolation and characterization of filamentous fungi from animal agricultural farm soil. Pertanika journal of science and technology, 25, 19-28.

[5] Silambarasan S \& Abraham J, (2013), Mycoremediation of Endosulfan and Its Metabolites in Aqueous Medium and Soil by Botryosphaeria laricina JAS6 and Aspergillus tamarii JAS9. PLoS ONE, 8(10).

[6] Ghosh A, Dastidar MG, \& Sreekrishnan TR, (2017), Bioremediation of chromium complex dyes and treatment of sludge generated during the process. International Biodeterioration \& Biodegradation, 119(Supplement C), 448-460.

[7] Korabecna M, (2007), The variability in the fungal ribosomal DNA (ITS1, ITS2, and 5.8 S rRNA gene): its biological meaning and application in medical mycology. Communicating current research and educational topics and trends in applied microbiology, 2, 783787.

[8] Dong T, Wu GW, Wang XN, Gao JM, Chen JG, \& Lee SS, (2010), Microbiological transformation of diosgenin by resting cells of filamentous fungus Cunninghamella echinulata CGMCC 3.2716. J. Mol. Catal B: Enzym, 67(3-4), 251-256.

[9] Haller MY, Müller SR, McArdell CS, Alder AC, \& Suter MJF, (2002), Quantification of veterinary antibiotics (sulfonamides and trimethoprim) in animal manure by liquid chromatography-mass spectrometry. Journal of Chromatography A, 952(1-2), 111-120.

[10] Jewell KS, Castronovo S, Wick A, Falås P, Joss A, \& Ternes TA, (2016), New insights into the transformation of trimethoprim during biological wastewater treatment. Water Research, 88, 550557.

[11] Perez C, Agnese AM, \& Cabrera JL, (1999), The essential oil of Senecia graveolens (composite): Chemical composition and antimicrobial activity tests. Journal of Ethnopharmacology, 66(11), 91-96.

[12] Jrah H, Kouidhi B, Flamimi G, \& Bakhrouf AM, T., (2011), Chemical composition, antimicrobial potential against cariogenic bacteria and cytotoxic activity of Tunisian Nigella sativa essential oil and thymoquinone. Food Chemistry, 129(4), 1469-1474.

[13] Wang JR \& Zhang LY, (2006), Simultaneous determination and identification of furazolidone, furaltadone, nitrofurazone, and nitrovin in feeds by HPLC and LC-MS. Journal of liquid chromatography \& related technologies, 29(377-390).

[14] Levison ME \& Levison JH, (2009), Pharmacokinetics and pharmacodynamics of antibacterial agents. Infectious disease clinics of north America, 23(4), 791-815.

[15] Dariush N, (2008), Effects of aeration, agitation and $\mathrm{pH}$ on the production of mycelial biomass and exopolysaccharide from the filamentous fungus Ganoderma lucidum. Dynamic Biochemistry, Process Biotechnology and Molecular Biology, 2(1), 14-18.

[16] Technologies A, Using DMSO as injection solvent to increase sample load in preparative LC. 2005: USA.

[17] Nouws JFM, Vree TB, Aerts MML, Degen M, \& Driessens F, (1987), Some pharmacokinetic data about furaltadone and nitrofurazone administered orally to preruminant calves. Veterinary Quarterly, 9(3), 208-214. 
[18] European Food Safety Authority E, (2015), Scientific Opinion on nitrofurans and their metabolites in food. European Food Safety Authority Journal, 13(6), 4140.

[19] Boelsterli UA, Ho HK, Zhou S, \& Leow KY, (2006), Bioactivation and hepatotoxicity of nitroaromatic drugs. Current Drug Metabolism, 7, 715-727.

[20] Aracena P, Lazo-Hernandez. C., Molina-Berrios A, Sepulveda DR, Reinoso C, Larrain JI, Navarro J, \& Letelier ME, (2014), Microsomal oxidative stress induced by NADPH is inhibited by nitrofurantoin redox biotransformation. Free Radical Research, 48, 129-136.

[21] Belinda SH, Christopher B, \& Shane RW, (2011), Nifurtimox activation by Trypanosomal Type I Nitroreductases generates cytotoxic nitrile metabolites. Journal of Biological Chemistry 286(15), 13088-13095.

[22] Ertan G, Karasulu Y, \& Güneri T, (1993), Degradation and gastrointestinal stability of nitrofurantoin in acidic and alkaline media. International Journal of Pharmaceutics, 96(1), 243-248. 